\title{
Wood Anatomy as a Link between Botany and Forestry*
}

\section{By B. J. RendLE}

$\mathrm{T}$ $\mathrm{HE}$ conception of forestry as being ultimately dependent for its advance upon the interaction of a number of more or less independent basic sciences is a familiar one. The problems associated with the tree considered as a plant and with the forest considered either as an ecological unit or an artificial crop call for special methods of attack which, while primarily concerned with some aspect of forestry, can incidentally contribute to the general fund of botanical knowledge. The study of wood anatomy, with special reference to the production and utilisation of timber, is a case in point. In modern times this somewhat specialised subject has advanced along several different lines according to the points of view of the investigators concerned. The demand for practical methods of identifying and investigating the physical properties of commercial timbers has stimulated the study of species in this category. This has naturally been carried out more with reference to the important timbers of certain geographical regions than with botanical groups, and the woods of the north temperate zone in particular have received more than their fair share of attention.

In Germany, long before the end of the last century, certain of the timber-producing species of outstanding importance to Continental forestry practice were submitted to intensive studies of their anatomical-technical characters, which, besides yielding results of direct practical application, were of value in instigating further research in the peculiar problems of plant physiology which are associated with the great size and long life of trees. More recently, the opening up of botanical exploration in the tropics has offered a wide field of investigation in wood anatomy. Numbers of new genera and species of great potential value as a source of timber incidentally provide material for the study of systematic anatomy which for richness and variety is far superior to the limited and rather specialised arborescent flora of the north temperate regions.

Botanical exploration in an entirely different direction, geological rather than geographical, has also been the means of advancing our knowledge of the structure of wood, particularly as regards the gymnosperms. Study of the structure of fossil plants has been of the greatest importance in the development of the subject; it has even been observed that at one time woody plants were better known anatomically in a fossil than in a living condition, and it is safe to say that some of the most valuable studies of coniferous woods have been prompted by a desire to elucidate the relationship of their fossil allies and their connexion with recent forms. These researches have

- From a paper read before a joint meeting of Sections K (Botany) and $K^{*}$ (Forestry) of the British Association, at York, on Sept. 5 .

No. 3292, VoL. 130] resulted in the formulation of criteria capable of wide application, and in this way palæobotany has probably had a stronger influence than either taxonomy or physiology on the progress of wood anatomy.

In considering the systematic value of wood characters, as with any other part of a plant, the first essential is to recognise the kinds of characters that are to be found. Different writers have divided characters into various classes and sub-classes, each with a more or less descriptive name, but for the present it is only necessary to refer to the two main divisions, namely, those which can be regarded as adaptations to environment, that is, 'biological characters', and those which have no apparent relation to the environment and remain unaffected by variations in the conditions of growth ; for these the term 'inherent characters' seems to be the most appropriate.

It is abundantly clear that the anatomical structure of wood is a product of two factors, heredity and adaptation, and the successful employment of anatomical characters for systematic purposes depends on our being able to distinguish constant, inherited characters from those which are due to the influence of environment. In the course of generations of research, botanists have arrived at a fair idea of what morphological characters are constant, and to what extent they can be interpreted as indicating relationships. But in the case of wood there is relatively little information on this point and the need for further work of this kind is most pressing. The reason for this is not far to seek. The plants which are the subject of investigation in most fields of applied biology are generally available in a more or less complete condition, or at the least, their naming and classification is based on a study of those parts which are easily obtainable, but in the case of trees the difficulty is that the plant is such a large object; it is a laborious undertaking to examine the complete range of morphological and anatomical variation in a single tree, and when it becomes necessary to take into account the further variations induced by different conditions of growth, the problem assumes a much more formidable aspect.

Systematic botanists who have had to concern themselves with the critical study of trees from herbarium specimens are familiar with the morphological variation which occurs and which has often led to two or more specific epithets being attached to the same species when the trees have been described and named on incomplete botanical material. The similar variation in the structure of wood has not been sufficiently realised by all anatomists and this has in turn resulted in descriptions and keys, based on a study of inadequate material and on characters 
which are not specific at all but only due to different conditions of growth, being used to differentiate between species. Many of the early researches in wood structure were directed towards the study of the size of the wood elements and their distribution within the growth ring, chiefly for purposes of identification. It is not yet fully realised in certain quarters that these features vary from tree to tree and that they vary more widely in different parts of the same tree.

Now, although this variation is considerable, the position of affairs is not so hopeless as it might appear at first sight. It will not be necessary to cut and examine microscopically the structure of hundreds of sections from dozens of different trees in order to obtain a reasonably accurate picture of the typical wood structure of every species. But it is necessary to examine in detail a few species exemplifying selected types of structure in order to ascertain the limits of variability of each diagnostic feature and to discover if possible the laws governing such variation. Critical anatomical surveys of this kind can only be undertaken by institutions in a position to obtain carefully selected trees and to examine them according to an ordered system of routine.

At the Forest Products Research Laboratory at Princes Risborough, the main consideration which underlies investigations in wood structure is naturally the influence of structure on the technical properties of the timber. There is no need to labour the point that in technological research one of the first essentials is to discover as much as possible about the physical nature of the material concerned. The cellular structure of wood is one of the most important factors in determining its physical properties, and a close correlation exists between changes in structure and changes in technical properties. The variation in the properties of timber grown under different conditions of climate, soil and sylviculture is one of the big disadvantages of the use of wood in industry for purposes which are in any degree exacting. The problem before us is to investigate the structure of a sufficient quantity of each species, grown under different conditions and in different parts of the country, so as to obtain an idea of the normal range of variation that is likely to occur. The same timber that is examined anatomically in the Laboratory is also subjected to technical tests. In this way its general quality is correlated with the growth features, thus indicating to the forester the conditions of growth necessary to produce first quality timber. At the same time, the results of these investigations are the means of increasing our knowledge of the general principles underlying the relation between the structure and the properties of wood, knowledge which is in demand for assessing the technical value of wood from an examination of its structure and for the proper understanding of the peculiar behaviour of wood under varying conditions of temperature, humidity and so on.
The lines of research that have been briefly outlined above, although directed primarily towards the solution of economic problems, are incidentally of importance in increasing our knowledge of the systematic anatomy of wood, and by virtue of their somewhat intensive character they are of special value in enabling us to distinguish between inherent and biological characters. From the severely practical point of view, this is of obvious importance in order to place the identification of timber specimens on a sound scientific basis, and there is ample evidence that the anatomical characteristics of wood can usefully be taken into account in the solution of taxonomic problems. There can be no serious suggestion of attempting a natural classification of woody plants on the sole basis of their vascular anatomy, but on the other hand there is no gainsaying the fact that the nearest approach to the ideal system of classification will be based on a complete knowledge of the external morphology and internal anatomy of plants. Wood anatomy is essentially auxiliary to taxonomy but it is an auxiliary that botanists cannot afford to neglect; it is, for example, of special value in throwing light on the systematic position of anomalous genera and provides a valuable test of the homogeneity of a family.

From the very nature of the subject the study of wood anatomy is confined to a comparatively small number of specialists, most of whom have been led thither by way of forestry or forest products research. In the belief that mutual benefit would result from some measure of organised co-operation between workers in different parts of the world, the opportunity offered by the Fifth International Botanical Congress at Cambridge in 1930 was taken by a few interested parties to discuss ways and means of effecting such co-operation. Eventually an International Association of Wood Anatomists was formed, which has as its object the advancement of the knowledge of wood anatomy in all its aspects; this Association seeks to achieve its object by enabling workers in the same field to get in touch with each other, by facilitating the exchange of ideas, information and material and by working towards standardisation in terminology and methods of description.

Co-operative work has already been started on the preparation of a polyglot glossary of terms used in describing woods. Anyone who has had occasion to study the recent literature of the subject in different languages will have noticed the lack of uniformity in the use of terms and the need for some measure of standardisation. As a first step in this direction a provisional glossary of terms has been compiled in six languages, and by circulating this repeatedly to experts in different countries for corrections and suggestions it is hoped in course of time to achieve an approach to finality.

What is perhaps a still more important undertaking is the effort to increase the amount of

No. 3292, Vol. 130] 
authentic material available for research. This can only be done by inducing collectors to obtain wood samples from the trunks of the same trees from which herbarium material is taken. It should be our aim to build up wood collections comparable to our national herbaria; those that we have are hopelessly encumbered by com. mercial samples of timbers and specimens col- lected in all good faith but entirely without regard to the need of ensuring their true botanical authenticity. There can be no better example of the need for co-operative effort than the preliminary work necessary to establish on a sound basis the thorough investigation and successful utilisation of the timber resources of the overseas dominions of the British Empire.

\section{Stellar Radial Velocities}

\section{By Dr. R. O. ReDman}

THERE has recently been published by the Lick Observatory a General Catalogue of the Radial Velocities of Stars, Nebulæ, and Clusters by Joseph Haines Moore.* This gives all radial velocities published up to January 1, 1932, and includes 6739 stars, 133 gaseous nebulæ, 18 globular clusters and 90 extra-galactic nebulæ. For some time a need has been felt for such a compilation, and after consultation with the members of the radial velocity commission of the International Astronomical Union, which includes practically all the workers active in this field, Dr. Moore undertook the task. Himself a radial velocity observer of considerable experience, he has carried out this work very thoroughly and the publication forms a compact collection of observational data of extremely great value.

It is very fitting that this catalogue should be issued by the Lick Observatory, which has played. such a leading part during the past forty years in the measurement of radial velocities of stars and nebulæ. The first attack on the measurement of the Doppler shift in stellar spectra was made by Huggins in 1866, using visual methods, but the task proved difficult, and reasonably reliable results were not obtained until Keeler's work with the Lick 36-inch refractor about 1890. Even then velocities were obtained only with one or two of the brightest stars in the sky.

However, at about this same time, line of sight velocities were successfully measured at Potsdam by photography. The great superiority of the photographic method was quickly realised and soon extensive work was started, particularly by Campbell and his associates at the Lick Observatory. Observations have been accumulating with increasing rapidity ever since. As is well known, the results have been among the most valuable of astrophysical data and have found many applications, in the determination of masses in binary stars, in the study of variable stars, in stellar motions and the dynamics of the Milky Way system. Radial velocity technique has also contributed support to relativity theory in observations of Sirius B and to the theory of the expanding universe in recent observations of the spiral nebulæ.

The practical problem of radial velocity determination consists in the measurement of displacements of lines in an absorption spectrum

* Publications of the Lick Observatory, Volume 18, 1932. No. 3292, Vol. 130] formed by light from an extremely faint source. The unavoidable complications present in all astronomical observations, namely a rotating earth and an unsteady atmosphere of varying transparency, have also to be contended with. The average stellar velocity in the line of sight is around 15 or $20 \mathrm{~km}$./sec., which corresponds in the ordinary photographic region of the spectrum to a Doppler shift of approximately one quarter of an angstrom.

The experience of the past thirty or forty years has indicated with little ambiguity the most desirable type of instrument to use. A telescope of large aperture is essential- -36 in. is the least size with which any important amount of work is done at present-and, although refractors have in several cases given excellent service, it is generally considered that reflectors are more desirable. Their advantages lie in ease of construction, in their perfect achromatism, in their greater efficiency in the region of shorter wavelengths and in the long equivalent focal length which may be obtained without an inconveniently long telescope tube. The Cassegrain arrargement of mirrors, with a convex secondary reflecting the beam back through a hole in the centre of the primary, is undoubtedly the best.

The spectrograph is normally carried on the telescope, and since it may then be required to work in almost any position, it has to be constructed and mounted with special precautions against flexure or distortion of any kind. Prisms are usually employed since they are less wasteful of light than are diffraction gratings. One prism is most commonly used, although the number may be two or three for the brighter stars, and several interchangeable cameras of various focal lengths are usually available. In this way a fairly wide range of dispersions can be provided, varying perhaps from near 10 to around 100 angstroms per millimetre at $\mathrm{H}_{\gamma}(\lambda$ 4340) and suitable for use with stars of a wide range of apparent brightness. Since effectively the observing is done in the open air, the spectrograph should be enclosed in an insulating case and its temperature controlled by a thermostat. Exposure times vary enormously with magnitude and type of stars, with observing conditions and with the particular instrument used. Extensive radial velocity work is rarely carried out where more than one or two hours per photograph are required in fair weather. The 